\title{
METODOLOGÍA PARA ELABORAR UN PLAN DE MEJORA CONTINUA
}

\section{METHODOLOGY FOR PREPARING A CONTINUOUS IMPROVEMENT PLAN}

\author{
Diana Ximena Proaño Villlavicencio ${ }^{1}$ \\ Victor Gisbert Soler ${ }^{2}$ \\ Elena Pérez Bernabeu ${ }^{3}$
}

1. Graduada en Ing. Industrial en la Universidad de Cuenca en la ciudad de CuencaEcuador, con dos años de experiencia en el sector administrativa. Master en Ingeniería de Organización y Logística en la Universidad Politécnica de Valencia (España). E-mail: xiprvil@epsa.upv.es

2. Doctor Ingeniero Industrial. Departamento de Estadística e Investigación Operativa Aplicadas y Calidad. Universidad Politécnica de Valencia (España). E-mail: vgisber@eio.upv.es

3. Doctor Ingeniero en Organización Industrial. Departamento de Estadística e Investigación Operativa Aplicadas y Calidad. Universidad Politécnica de Valencia (España). E-mail: elpeber@eio.upv.es

\section{Citación sugerida:}

Proaño Villavicencio, D.X. (2017). Metodología para elaborar un plan de mejora continua. 3C Empresa: investigación y pensamiento crítico, Edición Especial, 50-56. DOI: <http://dx.doi.org/10.17993/3cemp.2017.especial.50-56/>. 


\section{RESUMEN}

El presente artículo desarrolla el tema titulado "Metodología para Elaborar un Plan de Mejora Continua", este trata de un conjunto de acciones planeadas, organizadas, integradas y sistematizadas para obtener cambios, y mejoras de procedimientos en la organización.

La metodología consta de cinco niveles: análisis de las causas que provocan el problema, propuesta y planificación del plan de mejora, implantación y seguimiento continuo, y finalmente una evaluación de toda la metodología aplicada; estas acciones proporcionarán beneficios a la organización como reducción de costes, incremento de la productividad, mejora de la calidad, satisfacción del cliente, una adecuada comunicación entre los departamentos y mayor nivel productivo.

\section{ABSTRACT}

The present article develops the theme entitled "Methodology for Developing a Continuous Improvement Plan", which deals with a set of actions planned, organized, integrated and systematized to obtain changes and improvements in procedures in the organization.

The methodology consists of five levels: analysis of the causes that cause the problem, proposal and planning of the improvement plan, implementation and continuous monitoring, and finally an evaluation of the methodology applied; These actions will provide benefits to the organization such as cost reduction, increased productivity, improved quality, customer satisfaction, proper communication between departments and higher level of production.

\section{PALABRAS CLAVE}

Mejora continua, procesos, metodología, competencia, mercado.

\section{KEY WORDS}

Continuous improvement, processes, methodology, competition, market. 


\section{INTRODUCCIÓN}

Es normal que el ambiente empresarial este sujeto a competencias en el mercado, y a medida que su crecimiento es superior las empresas deben enfrentar nuevos retos por lo que deben ir mejorando en el transcurso del tiempo, a través de la aplicación de métodos de mejora continua, para poder superar sus debilidades y ser más competitivas en el entorno.

El plan de mejora es un proceso que se utiliza para alcanzar la calidad total y la excelencia de las organizaciones de manera progresiva, para así obtener resultados eficientes y eficaces. El punto clave del plan de mejora es conseguir una relación entre los procesos y el personal generando una sinergia que contribuyan al progreso constante.

La principal contribución de esta metodología sería el establecer cinco diferentes niveles, además indicar las conductas a seguir de cada uno de ellos logrando así el éxito en la implementación de la mejora continua.

\section{ANTECEDENTES}

Investigado varios libros y artículos se establece que:

Según (Barraza \& Dávila, 2008) La metodología para elaborar un plan de mejora continua se basa en la tercera esfera concéntrica del Kaizen dónde su propósito es eliminar el desperdicio buscando de esta manera la mejora de la calidad de los procesos y productos en un tiempo corto obteniendo resultados positivos y rápidos.

Pero el autor Rubert D'amelio (Damelio, 2001)nos habla sobre la importancia de la aplicación de diagramas de procesos para realizar el análisis entre cliente y proveedores, los cuales son base fundamental para la mejora.

Y en la ISO 9000 (Yánez \& Yánez, 2012) nos indica sobre la importancia de la mejora continua ya que hay gran demanda del cliente al exigir calidad en los productos, también nos muestra cómo implementar sistemas de mejora mediante auditorias, lo cual nos permite identificar los retos y oportunidades siendo estos factores de cambio y éxito de las organizaciones.

Se realizó una investigación sobre diferentes trabajos académicos donde recalcan la importancia en la aplicación de metodologías para la mejora continua en procesos, usando modelos de excelencia que son: el Enfoque de Gestión por Procesos y el Despliegue de la Función de Calidad, que se diseñaron con el fin de ofrecer a las organizaciones una metodología clara y fácil de mejora continua, que alcance los niveles de excelencia uniendo aspectos claves de cada uno de ellos. Nuñez también aplica los pasos que se explicaran en el presente artículo. (NUÑEZ, VÉLEZ, \& BERDUGO, 2004).

En otro artículo los autores Alayo Gómez Robert y Becerra Gonzales Angie (Becerra Gonzales \& Alayo Gómez, 2014) nos comenta sobre la implementación de un Plan de Mejora continua utilizando el círculo de Deming y las diferentes herramientas que se 
comentan en este artículo siendo estas: el diagrama de Pareto, diagrama causa efecto, AMFE, entre otros, de igual manera realiza los pasos descritos (análisis de las causas que provocan el problema, propuesta y planificación del plan, implantación y seguimiento, evaluación) pero los ejecuta de diferente manera.

\section{METODOLOGÍA}

La metodología a utilizar consiste en el análisis de las áreas a mejorar, definiendo los problemas a solucionar, y en función de estos estructurar un plan de acción, que esté formado por objetivos, actividades, responsables e indicadores de gestión que permita evaluar constantemente, este proceso debe ser alcanzable en un periodo determinado; y para ello el Plan de mejora deberá seguir los siguientes pasos:

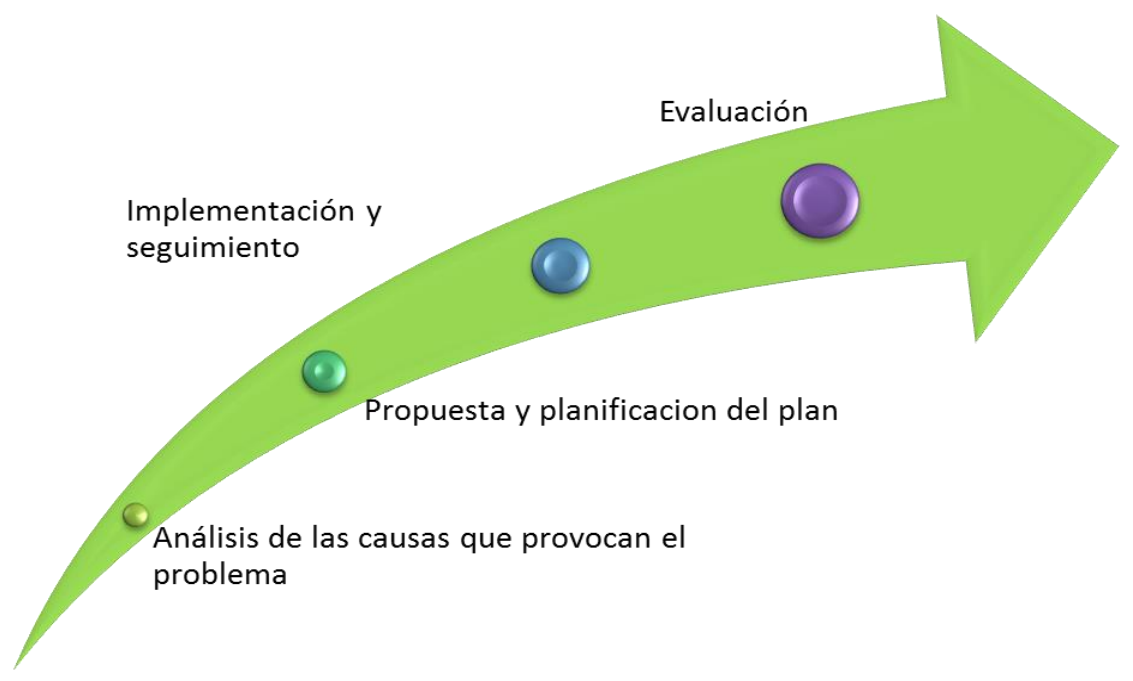

\section{Análisis de las posibles causas que han provocado problemas en el tiempo:}

Para cumplir con este requerimiento se recomienda tener en consideración los siguientes puntos.

- Identificar el área y procesos a ser mejorados, y para lo cual se debe ser priorizada en función de su importancia, en relación a la misión, visión y objetivos estratégicos de la organización.

- Analizar el impacto que tiene el proceso en el área, para alcanzar los objetivos estratégicos planteados por la empresa.

- Describir las causas y efectos negativos de la problemática, apoyándose con diversas herramientas y técnicas de análisis como:

Diagrama Causa- efecto (Espina de pescado). 
$\checkmark$ FODA (fortalezas, oportunidades, debilidades, amenazas)

$\checkmark$ Árbol del problema o Diagrama del árbol

$\checkmark$ Los 5 ¿ंPor qué?

$\checkmark$ AMFE (Análisis de Modo y Efecto de Falla)

\section{Propuesta y planificación del plan}

Deberá ser viable, flexible y que permita integrar nuevas acciones a corto, mediano o largo plazo, y para lo cual se debe tener en cuenta las siguientes acciones:

a) Definir objetivos y resultados del análisis realizado en el punto 1 .

b) Analizar las posibles soluciones apoyándose en herramientas como:

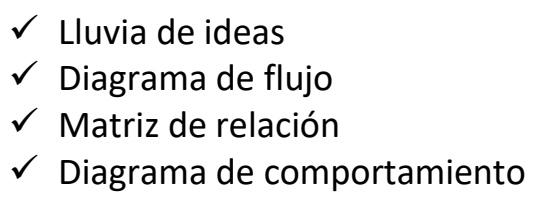

c) Establecer acciones para la solución: en esta fase es necesario asignar tareas a cada miembro del equipo; se sugiere aplicar diferentes herramientas para la solución de problemas que se han identificado, entre ellas están:
$\checkmark$ Planificación estratégica y operativa
$\checkmark$ Análisis y rediseño de procesos
$\checkmark$ Cuadro de mando integral
$\checkmark$ Benchmarking (Aprender Mejores Práctica)

d) Verificar la aplicación de las acciones en el proceso.

e) Especificar los indicadores que evidencien la mejora en el proceso.

f) Documentar el plan de mejora.

\section{Implementación y seguimiento.}

Para realizar la implementación y seguimiento del Plan de mejora es indispensable incorporar al proceso al personal encargado de realizar las acciones propuestas; los mismos que deben ejecutar las siguientes funciones:

- Informar sobre el plan.

- Ejecutar las acciones programadas con las personas involucradas

- Dar seguimiento en base a los indicadores de impacto y desempeño, este deberá realizarse en un periodo determinado por los involucrados.

- Verificar que se cumpla el plan de acuerdo a lo que se proyectó. 
- Valorar el cumplimiento del plan.

\section{Evaluación}

Este punto consiste en la verificar el cumplimiento del Plan de mejora continua de acuerdo a la propuesta, planificación e implantación.

La evaluación es necesaria para poder observar las irregularidades que han surgido en el tiempo de ejecución.

La técnica utilizada para la evaluación del Plan de mejora es:

- Diseñar un plan de evaluación, basándose en los objetivos e indicadores.

- Ejecutar el plan de evaluación.

- Realizar un informe sobre la evaluación indicando las ventajas y desventajas, de los resultados obtenidos del Plan de mejora.

\section{CONCLUSIONES}

El plan de mejora continua es una herramienta muy útil para las empresas que desean mejorar sus servicios, productos o procesos lo que les va a permitir permanecer en el mercado, crecer y ser competitivos. Su aplicación es muy útil y fácil e involucra a todos los niveles de la organización dependiendo del área o proceso a mejorar, lo importante para lograr los éxitos esperados en la aplicación de esta técnica es definir de manera exacta el área a mejorar, definiendo claramente los problemas a solucionar, y en función de estos estructurar el plan de acción a seguir definiendo objetivos claros, actividades, responsables e indicadores que permita evaluar el proceso de mejora todo esto dentro de un periodo determinado y bien definido. 


\section{REFERENCIAS BIBLIOGRÁFICAS}

Barraza, M. F. S., \& Dávila, J. Á. M. (2008). Encontrando al Kaizen: Un análisis teórico de la Mejora Continua. Pecvnia: Revista de la Facultad de Ciencias Económicas y Empresariales, Universidad de León(7), 285-311.

Becerra Gonzales, A. d. R., \& Alayo Gómez, R. D. (2014). Implementación del plan de mejora continua en el área de producción aplicando la metodología PHVA en la empresa Agroindustrias Kaizen.

Damelio, R. (2001). Mapeo de procesos: México, MX: Panorama Edit.

IsoTools. (2015). Cómo elaborar un plan de mejora continua. Retrieved from https://www.isotools.org/2015/05/07/como-elaborar-un-plan-de-mejoracontinua/

NUÑEZ, L., VÉLEZ, M., \& BERDUGO, C. (2004). Aplicación de una Metodología de Mejora de Procesos basada en el Enfoque de Gestión por Procesos, en los Modelos de Excelencia y el QFD en una empresa del sector de confecciones de Barranquilla. Revista Ingeniería y desarrollo(16), 45-58.

Pública, M. d. A. (2014). Guía para la elaboración e implementación de plan de mejora institucional. República Dominicana: Retrieved from http://map.gob.do/wpcontent/uploads/2012/04/GUIA-para-la-Elaboracion-e-Implementacion-del-Plande-Mejora-Institucional.pdf.

Pública, S. d. E. (2006). Guía para la elaboración del plan de mejora, grupos de mejora. México: Retrieved from https://es.slideshare.net/margaysabel/guia-para-laelaboracion-de-plan-de-mejora-para-instituciones-educativas.

Yánez, J., \& Yánez, R. (2012). Auditorías, Mejora Continua y Normas ISO: factores clave para la evolución de las organizaciones. Ingeniería Industrial. Actividad y Nuevas Tendencias, 83-92. g(IsoTools, 2015)

http://map.gob.do/wp-content/uploads/2012/04/GUIA-para-la-Elaboracion-eImplementacion-del-Plan-de-Mejora-Institucional.pdf(M. d. A. Pública, 2014) (S. d. E. Pública, 2006) 